\title{
Psychiatric sickness absence was affected by social support at home and at work
}

Stansfeld SA, Rael EGS, Head J, et al. Social support and psychiatric sickness absence: a prospective study of British civil servants. Psychol Med 1997 Jan;27:35-48.

\section{Objective}

To determine the effect of home and work related chronic stressors and social support on psychiatric sickness absence.

\section{Design}

Cohort study with 5 year follow up.

\section{Setting}

Civil service in London, England.

\section{Participants}

10308 London based civil servants between 35 and 55 years of age $(70 \%$ men) who worked in 20 non-industrial departments, completed questionnaires and had a physical examination. Analysis was done on 4202 participants (41\%) for whom the reason for sickness absence was available.

\section{Assessment of risk factors}

Social support (confiding and emotional, practical, and negative aspects) received from the person nominated by the participant as the closest to them was assessed using the Close Persons Questionnaire. Work social support was assessed by questions relating to feedback support from colleagues and supervisors and clarity and consistency of information from supervisors. Material problems were assessed using Pearlin's scale of chronic stressors.

\section{Main outcome measures}

Rates of short and long periods of psychiatric sickness absence per 100 person years, calculated separately for men and women.

\section{Main results}

High levels of negative social support from the closest person were associated with higher rates of sickness absence in men. The rate ratios (RRs) for the effect of high levels of negative support in men were $1.30,95 \%$ CI 1.03 to 1.64 for short absences and 2.52, CI 1.48 to 4.30 for long absences. The effect was not as clear in women. Confiding and emotional support was associated with higher rates of longer absences in women (RR 1.76 , CI 1.11 to 2.79 ) while medium levels of practical support were associated with lower rates of long absences in men (RR 0.57 , CI 0.33 to 0.98 ). Work social support was associated with lower rates of short absences in men and women (RR 0.73, CI 0.58 to 0.93 and 0.62 , CI 0.49 to 0.80 , respectively). The highest level of material problems was associated with increased rates of short and long absences in men (RR 1.44, CI 1.15 to 1.81 and 1.99 , CI 1.22 to 3.22 , respectively) and increased short absences in women (RR 1.45 , CI 1.12 to 1.88 ).

\section{Conclusions}

Negative aspects of social support and material problems increased psychiatric sickness absence. High levels of support at work were protective against short absences.

Source of funding: Institute for Work and Health, Toronto, Canada.

For article reprint:Dr S A Stansfeld, Department of Epidemiology and Public Health, University College London Medical School, 1-19 Torrington Place, London WC1E 6BT, UK. Fax $+44(0) 1718130242$.

\section{Commentary}

This useful study by Stansfeld et al provides relatively strong information about the effects of social support on psychiatric sickness absence. The authors included measures of various types of support and separated the results for men and women. The outcome measure was objective and verified in certain cases by contact with doctors. Consequently, although psychiatric sickness absence may not be frequent, compared with many physical health problems, it is clearly linked to social support.

Interestingly, negative support was shown to be linked to long absences in men, while confiding and emotional support were associated with increasingly long absences in women. These results show the importance of viewing the role of social support for men and women in a somewhat different light in clinical situations.

A central finding is that positive social support at work has a preventive effect on short term absences. This lends credence to a number of theoretical views, one of which is that stress at work may be mediated by social support. Above all, this finding has marked implications because social support at work may be amenable to interventions. Indeed, improving social support may be more practically achievable than changing other factors and the work setting may be an opportune place compared with other settings in which access and change are more difficult.

This study also highlights the complex way that various forms of positive and negative social support may promote or protect from psychiatric sick leave. For example, distinctions between negative and positive support by sex allow us to better understand how support works while at the same time they show that many factors determine the effect of social support.

Clinically, these findings suggest that social support is important and should probably be routinely assessed. Above all, the results underscore the need for clearer understanding of the mechanisms of social support at work because large differences were noted between types of support and sex. However, many of the ratios are rather small, and the results show that social support is but one part of a very complex outcome, namely psychiatric sickness absence.

Steven J Linton, PhD Örebro Medical Center Örebro, Sweden 